\title{
Relação entre o nível de atividade física e seus reflexos na saúde mental e na qualidade de vida da população durante a pandemia de COVID-19
}

\author{
Relationship between the level of physical activity and its reflections on the mental health and \\ quality of life of the population during the COVID-19 pandemic \\ Relación entre el nivel de actividad física y sus reflejos en la salud mental y la calidad de vida de la \\ población durante la pandemia del COVID-19
}

Recebido: 25/01/2022 | Revisado: 02/02/2022 | Aceito: 09/02/2022 | Publicado: 13/02/2022

Marcos Leão

ORCID: https://orcid.org/0000-0003-0136-1265 Centro Universitário Governador Ozanam Coelho, Brasil E-mail: markinleao93@gmail.com

Victor Neiva Lavorato

ORCID: https://orcid.org/0000-0001-9914-4722 Centro Universitário Governador Ozanam Coelho, Brasil E-mail: victor.lavorato@unifagoc.edu.br

Renata Aparecida Rodrigues de Oliveira

ORCID: https://orcid.org/0000-0002-5004-5253 Centro Universitário Governador Ozanam Coelho, Brasil E-mail: renata.oliveira@unifagoc.edu.br

Daniela Gomes Rosado

ORCID: https://orcid.org/0000-0003-4934-793X Centro Universitário Governador Ozanam Coelho, Brasil E-mail: daniela.rosada@unifagoc.edu.br

Isabela Alcântara Barretto Araújo Jardim ORCID: https://orcid.org/0000-0001-8691-5225

Universidade Federal de Ouro Preto, Brasil E-mail: isabela.araujo@aluno.ufop.edu.br

Mauro Cesar Isoldi

ORCID: https://orcid.org/0000-0003-4961-5332 Universidade Federal de Ouro Preto, Brasil E-mail: mauroisoldi@hotmail.com

Denise Coutinho de Miranda

ORCID: https://orcid.org/0000-0001-8714-6309

Universidade Federal de Ouro Preto, Brasil

E-mail: denisecmiranda@gmail.com

\begin{abstract}
Resumo
A diminuição dos níveis de atividade física, o aumento do estresse e a queda no convívio social estão diretamente relacionadas à redução da qualidade de vida. O objetivo do presente estudo foi verificar se o nível de atividade física afeta a qualidade de vida de adultos durante a pandemia da Covid-19. Foi realizado um estudo descritivo em indivíduos de ambos os gêneros, sendo aplicados três questionários, um que avalia o nível de atividade física (IPAQ), o outro que analisa a percepção subjetiva da qualidade de vida (WHOQOL) e um terceiro, que avalia a percepção do efeito da pandemia na qualidade de vida. Foi visto que 46,16\% dos participantes eram pouco ativos; aproximadamente $65 \%$ relataram piora na saúde mental e na qualidade de vida, sendo observadas alterações nos domínios físico, ambiental e social. Não foi possível relacionar a prática de atividade física com altos índices de qualidade de vida, mas observa-se que a pandemia influenciou negativamente a saúde mental e a qualidade de vida da população. Fazem-se necessários novos estudos e novas análises para melhor compreender essa relação entre a qualidade de vida e a atividade física em um cenário de pandemia.
\end{abstract}

Palavras-chave: Coronavírus; Atividade física; Qualidade de vida; Sedentarismo.

\begin{abstract}
The decrease in physical activity levels, the increase in stress and the drop in social life are directly related to the reduction of quality of life. The aim of the present study was to verify whether the level of physical activity affects the quality of life of adults during the COVID-19 pandemic. A descriptive study was carried out with individuals of both genders, with three questionnaires being applied, one that assesses the level of physical activity (IPAQ), the other that analyzes the subjective perception of quality of life (WHOQOL) and a third that assesses the perception of the effect of the pandemic on quality of life. It was seen that $46.16 \%$ of the participants were not very active; approximately $65 \%$
\end{abstract}


reported worsening in mental health and quality of life, with changes in the physical, environmental and social domains being observed. It was not possible to relate the practice of physical activity and good quality of life indices, but it is observed that the pandemic negatively influenced the mental health and quality of life of the population. New studies and new analyzes are needed to better understand this relationship between quality of life and physical activity in a pandemic scenario.

Keywords: Coronavirus; Physical activity; Quality of life; Sedentary lifestyle.

\section{Resumen}

La disminución de los niveles de actividad física, el aumento del estrés y la caída de la vida social están directamente relacionados con la reducción de la calidad de vida. El objetivo del presente estudio fue verificar si el nivel de actividad física afecta la calidad de vida de los adultos durante la pandemia de COVID-19. Se realizó un estudio descriptivo con individuos de ambos sexos, aplicándose tres cuestionarios, uno que evalúa el nivel de actividad física (IPAQ), otro que analiza la percepción subjetiva de la calidad de vida (WHOQOL) y un tercero que evalúa la percepción del efecto de la pandemia en la calidad de vida. Se vio que el 46,16\% de los participantes eran poco activos; aproximadamente el $65 \%$ informó empeoramiento en la salud mental y la calidad de vida, observándose cambios en los dominios físico, ambiental y social. No fue posible relacionar la práctica de actividad física y los índices de buena calidad de vida, pero se observa que la pandemia influyó negativamente en la salud mental y la calidad de vida de la población. Se necesitan nuevos estudios y análisis para comprender mejor esta relación entre calidad de vida y actividad física en un escenario de pandemia.

Palabras clave: Coronavirus; Actividad física; Calidad de vida; Estilo de vida sedentario.

\section{Introdução}

A pandemia relacionada ao Coronavírus Sars-CoV-2, causador da COVID-19, declarada pela Organização Mundial de Saúde, desde 11 de março de 2020 (WHO, 2020), vem gerando impactos negativos na saúde da população e na economia mundial (Gama \& Borges, 2020). Os governos do mundo todo têm adotado medidas para que diminua a propagação do vírus como: fechamento de escolas e faculdades, cancelamentos de eventos públicos, restrição de circulação e controles internacionais de viagem (IPEA, 2020).

De forma similar, o Brasil vive período de calamidade pública e restrições do Estado (IPEA, 2020). Isso tem afetado os sistemas de saúde, econômico e social, peças fundamentais na sociedade. Em detrimento à crise sanitária, o Estado foi pressionado a tomar atitudes que impedissem a propagação do vírus, dentre elas o isolamento social, regulamentado pelas portarias $n^{\circ} 356 / 2020$ e $n^{\circ}$ 54/2020 (Brasil, 2020a; Brasil, 2020b), e o distanciamento social, recomendado pelo Conselho Nacional de Saúde e regulamentado pela portaria No 1.565/2020 (Brasil, 2020c).

Em se tratando de uma epidemia global, com um vírus que possui um alto índice de transmissibilidade e considerável taxa de letalidade, a saúde psicológica dos indivíduos acaba sendo severamente afetada. Estima-se que de um terço a metade da população possa apresentar consequências psicológicas e psiquiátricas caso não receba cuidados adequados (Lima, 2020). Destaca-se o fato de que a saúde mental e o bem-estar psicológico tendem a ser negligenciados em tempos de pandemias (Schmidt et al., 2020).

No que tange a qualidade de vida, não existe apenas uma definição ou apenas uma palavra que resuma esse termo, pois existem inúmeras variantes que interferem diretamente na saúde do indivíduo como: qualidade dos serviços públicos, bem-estar emocional, conforto, moradia, relações pessoais e sucesso profissional - pontos que cada pessoa considera importante para si e para uma boa vivência em sociedade (Fleck et al., 2000; Pereira, 2005).

Quando se refere à qualidade de vida, é fundamental citar quais são os pilares básicos que existem dentro desse termo, chamados de "domínios da qualidade de vida", que são: Domínio Físico, que se refere ao estudo do corpo e a uma melhor compreensão sobre suas funções fisiológicas, como dor, qualidade do sono e repouso; Domínio Psicológico, que busca entender quais são as maneiras de pensar, de agir, de se expressar, de absorver emoções e quais são os reflexos no psicológico do indivíduo; Domínio das Relações Sociais, que reflete na capacidade de se relacionar com as pessoas e na maneira que o indivíduo se porta 
em sociedade; Domínio do Meio Ambiente, que consiste no meio físico em que o indivíduo está inserido, como segurança pública, transporte de qualidade e recursos financeiros (Fleck et al., 2000; Pereira, 2005).

Entre esses domínios e definições citados acima existe uma relação muito próxima com o tema estudado. O domínio físico é um fator altamente afetado pelo sedentarismo (Fleck et al., 2000). Com a pandemia, pessoas estão cada vez menos ativas fisicamente, em reflexo das leis de restrição. O domínio psicológico é afetado devido ao receio de se contrair o vírus, à instabilidade financeira, tendo em vista o aumento do desemprego e falências de empresas, à restrição da liberdade de circulação e à influência dos meios de comunicação, que a todo momento divulgam notícias ruins. O domínio social também é extremamente afetado, devido a novas regras de circulação, restrição de qualquer tipo de relação social, cancelamento de shows, festas e eventos. E, por fim, o aspecto ambiental também sofre alterações, devido ao novo padrão da sociedade. Portanto, a queda na qualidade de vida está diretamente associada aos fatores supracitados (Maqsood et al., 2021).

Sabe-se que a prática de exercício físico melhora a qualidade de vida (Silva et al., 2010). Porém, tendo em vista que as medidas restritivas impostas na sociedade impossibilitam qualquer tipo de aglomeração e que as academias de ginástica, centros de treinamento, clubes de esporte/lazer, quadras de esportes coletivos estão fechados ou temporariamente fechadas no contexto da Covid-19, a prática regular de exercícios físicos torna-se limitada (Crochemore-Silva et al., 2020).

Além disso, a prática regular de exercícios físicos ajuda no combate a doenças cardiovasculares, dislipidemia e obesidade, que podem agravar o quadro do indivíduo que contrai o vírus Sars-CoV2 causador da Covid-19 (Rajpal, Rahimi \& Iasmil-Beigi, 2020). Portanto é importante incentivar a prática de exercícios mesmo em tempos de pandemia, como uma medida de precaução, fazendo com que a população mantenha uma rotina de exercício regular, principalmente idosos que fazem parte do grupo mais afetado (Polero et al., 2021). O ato de se manter ativo estabelece relação direta com o sistema imunológico, por exemplo, auxiliando na diminuição dos impactos da doença no organismo e ajudando na prevenção do agravamento do quadro (Ferreira et al., 2020).

Sabendo que antes da pandemia os índices de obesidade, depressão e ansiedade já preocupavam os especialistas da área de saúde (Ferreira et al., 2019), com a prática do distanciamento social é possível prever um agravamento dessa situação e consequentemente uma piora na qualidade de vida (Park et al., 2021). Dessa forma, faz-se necessário realizar novos estudos a fim de verificar a percepção da qualidade de vida associado ao nível de atividade física durante a pandemia do Sars-Cov2.

Portanto, o objetivo do presente estudo foi verificar se o nível de atividade física afeta a qualidade de vida de adultos durante a pandemia da Covid-19.

\section{Metodologia}

Foi realizado um estudo descritivo com delineamento transversal. A mostra foi selecionada por conveniência, sendo assim a população do estudo foi composta por 65 indivíduos de ambos os sexos, com idade de 18 a 60 anos, praticantes ou não de exercícios físicos. A coleta de dados foi feita através de um questionário on-line, produzido no Google Forms ${ }^{\circledR}$ e enviado aleatoriamente para os voluntários por meio do aplicativo WhatsApp ${ }^{\circledR}$. Para confirmação do interesse em participar voluntariamente, foi aplicado um Termo de Consentimento Livre e Esclarecido aos participantes do estudo, obedecendo às normas para a realização de pesquisas em seres humanos, Resolução no 466/12 e Resolução no 510/16 do Conselho Nacional de Saúde. Foram excluídos do estudo aqueles que não concordaram com o termo de consentimento livre e esclarecido ou não responderem a todas as perguntas dos questionários.

A princípio, foi utilizado um questionário contendo 14 perguntas, a fim de avaliar o efeito da pandemia da Covid-19 sobre a prática de atividades físicas regulares e na percepção da qualidade de vida (adaptado de Estima et al., 2020). Esse questionário tinha o objetivo de avaliar quais padrões comportamentais foram alterados devido a pandemia e quais seriam os possíveis impactos na sociedade com essa nova forma de viver. 
Além disso, para avaliar o nível de qualidade de vida do indivíduo, foi utilizado o Instrumento de Avaliação de Qualidade de Vida da Organização Mundial da Saúde (WHOQOL - bref). O questionário é composto por 26 perguntas; as perguntas número 1 e 2 versam sobre a qualidade de vida geral; as demais compõem 4 domínios: físico, psicológico, relações sociais e meio ambiente. As respostas seguem uma escala de Likert (de 1 a 5 , quanto maior a pontuação, melhor a qualidade de vida), sendo necessário recodificar o valor das questões 3,4 e $26(1=5),(2=4),(3=3),(4=2)$ e $(5=1)$. Em cada faceta foram somados os valores da entrevista e o resultado foi dividido pelo número de participantes, obtendo-se uma média em que o resultado foi de 1 a 5 (Fleck et al., 2000).

Também foi utilizado o Questionário Internacional de Atividade Física (IPAQ - versão curta) como instrumento para mensurar o nível de atividade física praticado pelos indivíduos. O questionário era constituído por 4 perguntas, sendo cada uma composta por letras A e B. As perguntas estão relacionadas ao tempo que a pessoa gastou fazendo atividade física na última semana (Matsudo et al., 2001). Os termos de classificação do questionário se caracterizam em sedentário, irregularmente ativo A e B, ativo e muito ativo, de acordo com o próprio IPAQ (Matsudo et al., 2001).

Os dados foram apresentados como média e desvio padrão da média ou porcentagem simples. Inicialmente, foi realizado um teste para avaliar a normalidade dos dados de Shapiro-Wilk. Posteriormente, foi realizado um teste t de Student para comparação entre os grupos praticante e não praticante de atividade física. Foi utilizado também o teste ANOVA de uma via com post hoc de Tukey para comparar os domínios de qualidade de vida de acordo com as classificações da autopercepção da qualidade de vida. O nível de significância adotado foi de 5\%. Os dados foram analisados através do programa estatístico GraphPad Prism $8.0^{\circledR}$.

\section{Resultados}

A Tabela 1 apresenta os dados sobre idade e sexo dos participantes. A maior parte dos avaliados era solteiro e tinha de 18 a 25 anos. Apenas 3,1\% da amostra não divide casa. Cerca de 50\% dos voluntários não praticam atividade física.

Tabela 1. Dados gerais dos participantes do estudo. Ubá-MG, 2021.

\begin{tabular}{|c|c|c|c|c|c|}
\hline \multirow{2}{*}{ Sexo } & Feminino & Masculino & & & \\
\hline & $43,1 \%$ & $56,9 \%$ & & & \\
\hline \multirow[b]{2}{*}{ Estado civil } & Solteiro (a) & Casado (a) & Divorciado (a) & Viúvo $(a)$ & \\
\hline & $87,7 \%$ & $10,8 \%$ & $1,5 \%$ & $0 \%$ & \\
\hline \multirow[t]{2}{*}{ Faixa etária } & 18 a 25 anos & 26 a 30 anos & $\begin{array}{c}31 \text { a } 40 \\
\text { anos }\end{array}$ & 41 a 50 anos & Mais de 50 anos \\
\hline & $70,8 \%$ & $20,0 \%$ & $3,1 \%$ & $1,5 \%$ & $4,6 \%$ \\
\hline \multirow{2}{*}{ Divide casa? } & Não & 1 pessoa & 2 pessoas & 3 pessoas & 4 ou mais pessoas \\
\hline & $3,1 \%$ & $24,6 \%$ & $30,8 \%$ & $18,5 \%$ & $23,1 \%$ \\
\hline \multirow{2}{*}{$\begin{array}{c}\text { Pratica } \\
\text { atividade física } \\
\text { há mais de três } \\
\text { meses? }\end{array}$} & $\begin{array}{c}\text { Mais de } 3 \text { vezes } \\
\text { por semana }\end{array}$ & $\begin{array}{c}1 \text { a } 3 \text { vezes por } \\
\text { semana }\end{array}$ & $\begin{array}{c}\text { Não pratica atividade } \\
\text { física }\end{array}$ & & \\
\hline & $21,5 \%$ & $29,2 \%$ & $49,2 \%$ & & \\
\hline
\end{tabular}

Fonte: Dados da pesquisa.

O Gráfico 1 apresenta o nível do distanciamento social que está sendo orientado pelas autoridades de saúde, ou seja, ficar em casa e evitar contato com outras pessoas. Destaca-se o fato de que apenas 9,2\% da amostra cumpre "pouco" ou "muito pouco" as orientações de distanciamento. 
Gráfico 1. Nível de Distanciamento Social. Ubá-MG, 2021.

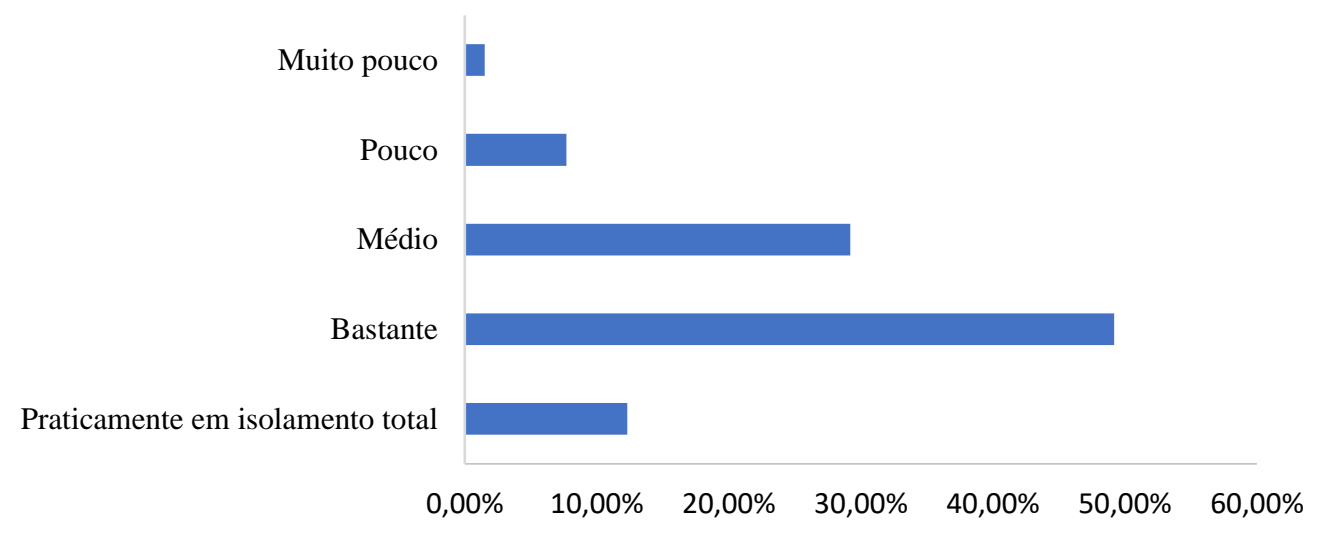

Fonte: Dados da pesquisa.

O Gráfico 2 apresenta os principais motivos pelos quais os participantes saem de casa. Apenas 9,20\% da amostra afirmou "fica em casa o tempo todo".

Gráfico 2. Principais motivos pelos quais os participantes saem de casa. Ubá-MG, 2021.

Sai todos os dias para alguma atividade

Sai todos os dias, o dia todo, para trabalhar ou atividade regular

Sai de vez em quando para fazer compras e esticar as pernas

Sai apenas para comprar o essencial, como comida e remédios

Fica em casa o tempo todo

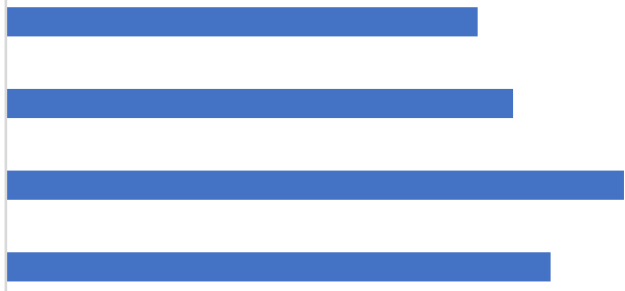

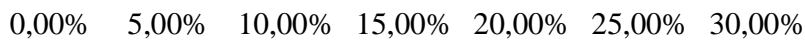

Fonte: Dados da pesquisa.

O Gráfico 3 apresenta as principais pessoas que frequentam a casa do participante.

Gráfico 3. Principais pessoas que frequentam a casa do participante. Ubá-MG, 2021.

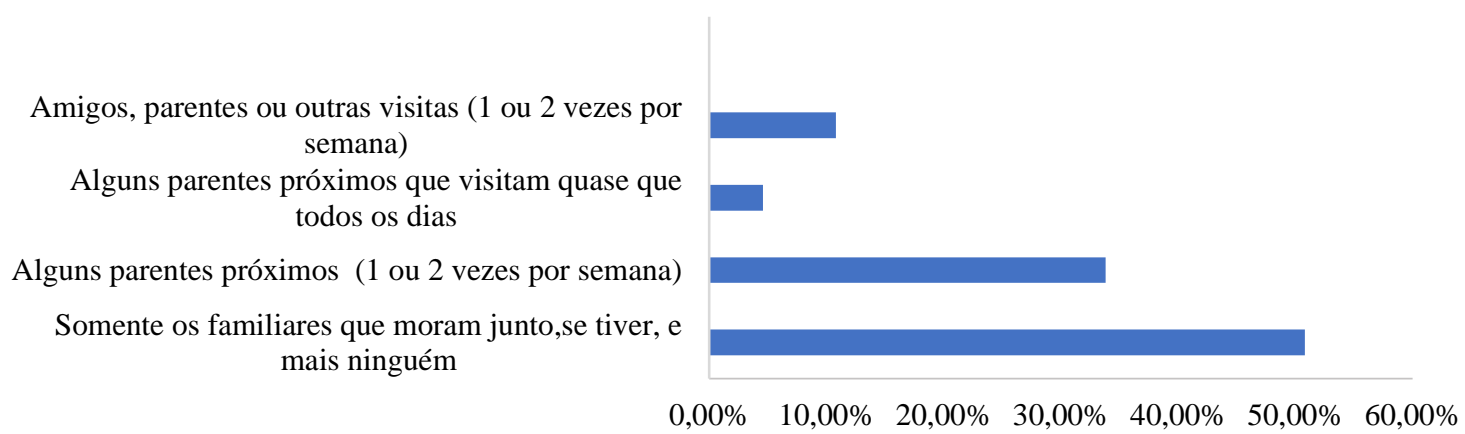

Fonte: Dados da pesquisa. 
O Gráfico 4 demonstra a autopercepção de como a saúde mental ficou prejudicada ao longo da pandemia. Verificouse que $64,60 \%$ dos voluntários afirmaram que a saúde mental piorou ao longo da pandemia, enquanto $16,90 \%$ acreditam que a saúde mental melhorou e 18,50\% não perceberam alteração.

Gráfico 4. Autopercepção da saúde mental ao longo da pandemia. Ubá-MG, 2021

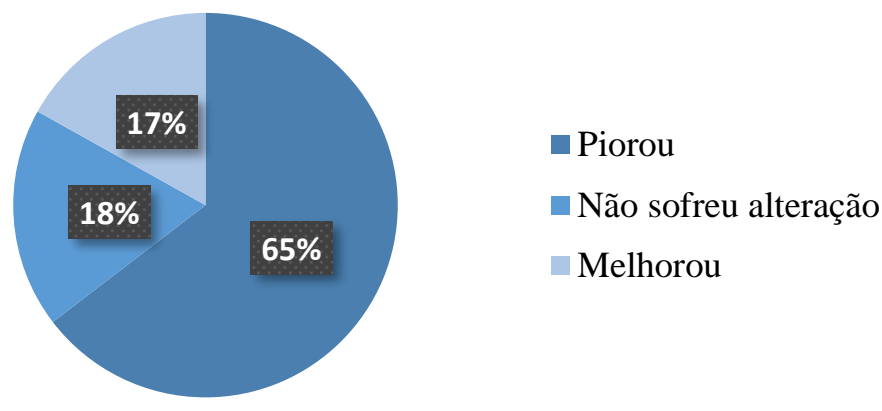

Fonte: Dados da pesquisa.

O Gráfico 5 apresenta a autopercepção de como a pandemia relacionada à COVID-19 influenciou na qualidade de vida.

Gráfico 5. Autopercepção de como a pandemia relacionada ao COVID-19 influenciou na qualidade de vida. Ubá-MG, 2021.

Está muito melhor do que antes da pandemia

Está melhor do que antes da pandemia

Está do mesmo jeito do que antes da pandemia

Está pior do que antes da pandemia

Está muito pior do que antes da pandemia

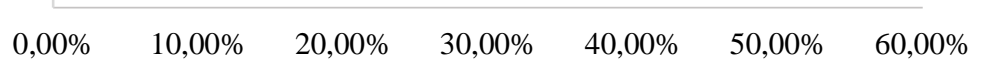

Fonte: Dados da pesquisa.

O Gráfico 6 demonstra a alteração na frequência do consumo de álcool, tabaco, remédios controlados ou outras drogas ao longo da pandemia relacionada à COVID-19. 
Gráfico 6. Alteração na frequência do consumo de álcool, tabaco, remédios controlados ou outras drogas ao longo da pandemia relacionada à COVID-19. Ubá-MG, 2021.

Não consome nada do que foi citado na questão

Não houve alteração
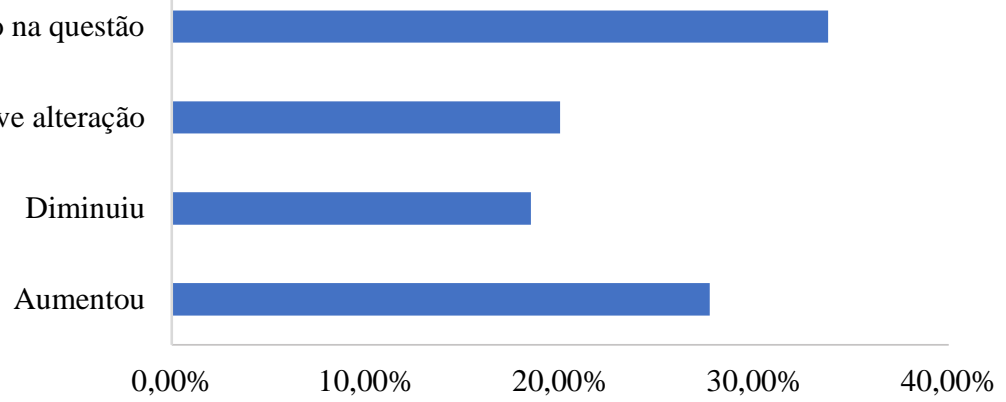

Fonte: Dados da pesquisa.

A Tabela 2 apresenta os dados relativos ao nível de atividade física dos avaliados. Destaca-se o fato de que 46,16\% foram classificados como "sedentário", "insuficientemente ativo B ou "insuficientemente ativo A".

Tabela 2. Nível de atividade física dos avaliados. Ubá-MG, 2021.

\begin{tabular}{cc}
\hline & Participantes (\%) \\
\hline Sedentário & $12,31 \%$ \\
Insuficientemente ativo B & $27,70 \%$ \\
Insuficientemente ativo A & $6,15 \%$ \\
Ativo & $22,5 \%$ \\
Muito ativo & $29,23 \%$ \\
\hline
\end{tabular}

Fonte: Dados da pesquisa.

A Tabela 3 mostra os dados relativos à qualidade de vida avaliada nos participantes. Os participantes foram agrupados em "pouco ativo", abrangendo a população classificada como "sedentário", "insuficientemente ativo B ou "insuficientemente ativo A" e "ativo", abrangendo os grupos classificados como "ativos" e "muito ativos". Não houve diferença estatística entre os grupos analisados $(\mathrm{p}>0,05)$.

Tabela 3. Domínios e Qualidade de vida total dos participantes avaliados. Ubá-MG, 2021.

\begin{tabular}{cccccc}
\hline & Físico & Psicológico & Social & Ambiental & $\begin{array}{c}\text { Qualidade de } \\
\text { Vida }\end{array}$ \\
\hline $\begin{array}{c}\text { Pouco } \\
\text { ativo }\end{array}$ & $48,33 \pm 11,28$ & $54,44 \pm 7,73$ & $46,39 \pm 18,14$ & $43,96 \pm 9,57$ & $48,28 \pm 5,60$ \\
\hline Ativo & $43,88 \pm 11,11$ & $55,24 \pm 7,38$ & $43,57 \pm 20,72$ & $44,91 \pm 7,91$ & $46,90 \pm 6,54$ \\
\hline
\end{tabular}

Fonte: Dados da pesquisa.

Os voluntários foram agrupados em três grupos de acordo com a autopercepção de como a pandemia relacionada à Covid-19 influenciou na qualidade de vida (dados provenientes do Gráfico 4). Os grupos foram: "Piorou" - pessoas que perceberam piora na qualidade de vida após a pandemia; "Neutro" - pessoas que não perceberam diferença na qualidade de vida após a pandemia; "Melhorou" - pessoas que perceberam melhora na qualidade de vida após a pandemia. Após agrupados, foram comparados os dados das dimensões e qualidade de vida total dos participantes avaliados (Tabela 4). Para o domínio físico, foi observado diferença estatística do grupo "piorou” para o "neutro", que apresentou maiores índices quando comparado ao grupo “neutro". Para o domínio social, o grupo "piorou” apresentou melhores índices quando comparado com o grupo "melhorou”. 
Por fim, o grupo "piorou" apresentou piores índices no domínio ambiental, quando comparado com os grupos "melhorou" e "neutro". Não se observou diferença estatística na qualidade de vida em geral.

Tabela 4. Domínio e Qualidade de vida total dos participantes avaliados de acordo com a autopercepção dos voluntários. UbáMG, 2021.

\begin{tabular}{cccccc}
\hline & Físico & Psicológico & Social & Ambiental & $\begin{array}{c}\text { Qualidade de } \\
\text { Vida }\end{array}$ \\
\hline Melhorou & $41,48 \pm 11,80$ & $57,37 \pm 4,20$ & $31,41 \pm 9.09$ & $50,00 \pm 6,62$ & $45,07 \pm 3,22$ \\
\hline Neutro & $38,21 \pm 9,53$ & $55,00 \pm 8,28$ & $41,67 \pm 15,71$ & $49,38 \pm 6,03$ & $46,06 \pm 5,03$ \\
\hline Piorou & $49,15 \pm 10,40^{*}$ & $54,07 \pm 7,38$ & $49,80 \pm 20,70^{\#}$ & $41,59 \pm 8,49^{* \#}$ & $48,65 \pm 6,78$ \\
\hline
\end{tabular}

*, Diferença para Neutro; \#, Diferença para Melhorou. Fonte: Dados da pesquisa.

\section{Discussão}

O objetivo do presente estudo foi verificar se o nível de atividade física afeta a qualidade de vida de adultos durante a pandemia da Covid-19. Os dados mostram que parte da amostra se autodeclara estar em alto grau de isolamento (61,5\%); 46,16\% dos voluntários foram considerados sedentário ou insuficientemente ativo; 65\% observaram piora na saúde mental; 64,6\% acreditam que a qualidade de vida piorou ao longo da pandemia, porém sem alteração da qualidade de vida geral, mas com alteração nos domínios físico, ambiental e social.

A maior parte dos entrevistados acredita estar cumprindo o distanciamento social, porém $41,5 \%$ afirmaram sair todos os dias, seja para trabalhar ou realizar algum tipo de atividade. Além disso, 49,2\% recebem visita em casa. Os dados sugerem que a prática de distanciamento social pode não estar sendo tão efetiva.

Destaca-se que um dos métodos mais eficazes para o combate à doença é o distanciamento social, que restringe a convivência em sociedade e consequentemente diminui os índices de transmissão do vírus. Tal medida é essencial para desacelerar a propagação do vírus em períodos epidemiológicos, em que a taxa de transmissão é extremamente alta (Garcia; Duarte, 2020), impedindo que o sistema de saúde não entre em colapso e possa atender a demanda da população até que ocorra uma vacinação em massa (Tonin et al., 2020).

Porém, o distanciamento social tem gerado alguns resultados negativos na população. $\mathrm{O}$ ato de adotar tais medidas ou o receio de se contaminar podem abalar a saúde mental e o bem-estar psicológico das pessoas (Ornell et al., 2020).

Como consequência disso, Baccarin e Oliveira (2021) observaram aumento no consumo de medicamentos ansiolíticos, bebidas alcóolicas e tabaco. Além disso, Gunnell et al. (2020) afirmam que estressores financeiros e uso de álcool e outras drogas tendem a se elevar em momentos de pandemia. Tal fato também foi observado em nosso estudo, no qual $27,70 \%$ dos voluntários relataram aumento no consumo de alguma dessas substâncias.

Sabe-se que a qualidade de vida possui diferentes domínios, os quais podem afetar o indivíduo positiva ou negativamente (Pereira et al., 2006). As relações sociais exercem um grande papel positivo dentro das vertentes da qualidade de vida. Com os novos métodos de convívio da sociedade, o ato de se relacionar ficou cada vez mais restrito e limitado. O fechamento de casas de eventos, o cancelamento de shows, o fechamento de museus e a proibição de festas culturais mostramse favoráveis para que as relações sociais diminuam e o número de pessoas sem essa vivência aumente (Minayo et al., 2000). Tal realidade pode explicar, pelo menos em parte, os baixos valores encontrados no domínio social para todos os grupos analisados.

Um importante fator que contribui positivamente para uma boa qualidade de vida é o nível de atividade física, que deve 
estar dentro do limite recomendável para manutenção da saúde. A OMS recomenda que adultos façam de 150 a 300 minutos de atividade moderada durante a semana e 75 a 150 minutos de atividade intensa, caso não haja nenhuma contraindicação. Um dos parâmetros também usado é do American College of Sports Medicine (ACSM), que implica que adultos mantenham pelo menos 30 minutos diários de atividade moderada, 5 vezes na semana, ou 20 minutos de atividade física de intensidade alta, 3 vezes na semana além das atividades cotidianas (Lima et al., 2014).

Relatos de melhoria na qualidade do sono, no humor e no bem-estar foram observados em praticantes de atividade física. Estar fisicamente ativo ajuda no enfrentamento da pandemia, amenizando os impactos de uma forma geral, seja dos problemas físicos ou psiquiátricos da população (Avelino, 2020).

No presente estudo, $46,16 \%$ dos voluntários foram considerados sedentário ou insuficientemente ativo. Atualmente, as pessoas estão mais propensas a apresentar comportamento sedentário, realizando atividades que exigem um baixo gasto energético. Segundo o estudo realizado por Malta et al. (2020), observou-se um aumento no número de pessoas fisicamente inativas, como consequência da pandemia da Covid-19.

Destaca-se que o sedentarismo é um dos principais fatores de risco para o desenvolvimento de doenças que agravam os sintomas da Covid-19, como doenças cardiovasculares, diabetes, hipertensão, dislipidemia e obesidade (Avelino et al., 2020). De acordo com Pereira et al. (2003), a obesidade já é uma epidemia mundial, independente das condições econômico-sociais. Tendo em vista que metade dos entrevistados não praticam nenhum tipo de atividade física, essa população pode apresentar risco para o acometimento da obesidade e consequente agravamento da doença.

Destacando a faixa etária, foi observado que a maioria da população avaliada está no período pós-adolescência, evidenciando um desinteresse cada vez mais cedo pela atividade física. É possível relacionar os altos níveis de inatividade física com as novas práticas de hobby dos anos de 2021, como plataformas digitais que disponibilizam vários serviços ao mesmo tempo, como as redes sociais, os jogos de entretenimento on-line, vendas e prestações de serviço. O mundo on-line em 2021 ficou mais popular e foi uma forma mais segura de prestação de serviços, evitando ao contato físico, que foi suspenso devido a pandemia.

As práticas de distanciamento social impostas pela pandemia restringiram o convívio social às plataformas digitais ou ao contato apenas de familiares mais próximos, conforme observado em nossa pesquisa, segundo a qual aproximadamente $50 \%$ dos participantes relataram conviver apenas com familiares mais próximos ou membros da casa. Dessa forma, observa-se que os indivíduos ficam cada vez mais sozinhos e que tanto as políticas de prevenção, quanto as de contenção do vírus, estão associadas com a falta de atividade física devido a limitação de circulação.

Todos os fatores supracitados contribuem para que haja uma queda na qualidade de vida do indivíduo. Esses dados corroboram com os nossos achados, segundo os quais $64,60 \%$ dos participantes afirmaram piora na autopercepção da saúde mental e 64,6\% destacaram piora na qualidade de vida. Estudos demonstram que as medidas restritivas, como a limitação de circulação de pessoas, podem agravar os quadros de depressão para pessoas pré-dispostas (Dias et al., 2014; Lima, 2020).

No presente estudo, foram analisados os domínios da qualidade de vida dos participantes avaliados. A princípio, os voluntários foram separados em dois grupos: "ativo" e "pouco ativo". Não foi observado diferença estatística. Apesar de o questionário WHOQOL-bref não possuir uma classificação específica, os valores apresentados na qualidade de vida geral são baixos, considerando que o instrumento pode atingir uma pontuação de até 100 pontos. Em nosso estudo, os valores foram menores que 50 pontos.

Em seguida, os voluntários foram separados em três grupos ("melhorou", "neutro" e "piorou"), considerando a autopercepção dos voluntários em relação à qualidade de vida. Curiosamente, foram observados maiores índices para os domínios ambientais e sociais dos voluntários que declararam piora na sua qualidade de vida em relação ao grupo que alegou melhoria. Além disso, também foram observadas melhores médias para os domínios físicos e ambientais do grupo "piorou” em 
relação aos voluntários que não observaram diferença ("neutros"). A qualidade de vida geral não diferiu entre os grupos. Apesar de não haver diferença estatística entre os grupos, o domínio psicológico pode ter influenciado na qualidade de vida geral, a qual, novamente, apresentou baixa pontuação.

\section{Considerações Finais}

Este estudo tentou demonstrar que a atividade física influencia na saúde mental e na qualidade de vida da população durante a pandemia de COVID-19. Porém, não foi possível relacionar a prática de atividade física com bons índices de qualidade de vida. Quando houve relação entre a percepção subjetiva da qualidade de vida e o WHOQOL-bref, foi visto que, para os domínios físico, ambiental e social, o grupo de voluntários que declararam piora na qualidade de vida apresentou melhores índices quando comparado com o grupo que declarou melhoria ou com o grupo que não percebeu alteração (neutro).

Apesar de os dados serem controversos, é possível notar que a pandemia influenciou negativamente a saúde mental e qualidade de vida da população. Fazem-se necessários novos estudos e novas análises para melhor compreender essa relação.

É importante salientar as limitações do presente estudo. O número de avaliados foi pequeno, o questionário foi aplicado de forma online e o estudo é transversal, o que pode influenciar a interpretação dos resultados.

\section{Referências}

Avelino, E. B., Morais, P. S. A., Santos, A. C. B. C., Bovi, A. C. N., Paz, N. H., Santos, A. L. S., \& Lima, J. H. M. (2020). Fatores de risco para doença cardiovascular em adultos jovens sedentários. Brazilian Journal of Development, 6(8), 58843-58854.

Baccarin, J.G., \& Oliveira, J.A. (2021). Inflação de alimentos no Brasil em período da pandemia da Covid 19, continuidade e mudanças. Segurança Alimentar e Nutricional., 28.

Barros, A. J. D., Victora, C. G., Menezes, A. M. B., Horta, B. L., Hartwig, F., Victora, G., Pellanda, L. C., Dellagostin, O. A., Struchiner, C. J., Burattini, M. N., Gonçalves, M. R., Possuelo, L. G., Weber, L. P., Estima, S. L., Härter, J., Silva, S. G., Frizzo, M., Lima, R. C., Barros F. C., Silveira, M. F., \& Hallal, P. C. (2020). Padrões de distanciamento social em nove cidades gaúchas: estudo Epicovid19 / RS. Revista de Saúde Pública, $54, .75$.

Brasil. Ministério da Saúde. Gabinete do Ministro. (2020a). Portaria n 356, de 11 de março de 2020. Brasília.

Brasil. Ministério da Saúde. Gabinete do Ministro. (2020b). Portaria nº 54, de 01 de abril de 2020. Brasília.

Brasil. Ministério da Saúde. Gabinete do Ministro. (2020c). Portaria n ${ }^{\circ}$ 1.565, de 18 de junho de 2020. Brasília.

Dias, P. J. P., Domingos, I. P., Ferreira, M. G., Muraro, A. P., Sichieri, R., \& Silva, R. M. V. G. (2014). Prevalência e fatores associados aos comportamentos sedentários em adolescentes. Revista de Saúde Pública, 48(2), 266-274.

Ferreira, A. P. de S., Szwarcwald, C. L., \& Damacena, G. N. (2019). Prevalência e fatores associados da obesidade na população brasileira: estudo com dados aferidos da Pesquisa Nacional de Saúde, 2013. Revista Brasileira de Epidemiologia, 221-14.

Ferreira, M. J., Irigoyen, M. C., Colombo, F. C., Saraiva, J. F. K., \& Angelis, K. D. (2020). Vida Fisicamente Ativa como Medida de Enfrentamento ao COVID19. Arquivos Brasileiros de Cardiologia, 114(4), 601-602.

Fleck, M. PA, Louzada, S., Xavier, M., Chachamovich, E., Vieira, G., Santos, L., \& Pinzon, V. (2000). Aplicação da versão em português do instrumento abreviado de avaliação da qualidade de vida "WHOQOL-bref". Revista de Saúde Pública, 34(2), 178-183.

Garcia, L. P., \& Duarte, E. (2020). Intervenções não farmacológicas para o enfrentamento à epidemia da COVID-19 no Brasil. Epidemiologia e Serviços de Saúde, 29(2).

Gunnell, D., Appleby, L, Arensman, E., Hawton, K., John, A., Kapur, N., Khan, M., C O'Connor, R., \& Pirkis, J.(2020). Suicide risk and prevention during the COVID-19 pandemic. Lancet Psychiatry.7(6), 468-47.

Instituto de Pesquisa Econômica Aplicada. (2020). 31: Instrumentos de políticas públicas para o enfrentamento do vírus da COVID-19: uma análise dos normativos produzidos pelo Executivo Federal. Brasília.

Lima, D.F., Levy, R.B., \& Luiz, O.C. (2014). Recomendações para atividade física e saúde: consensos, controvérsias e ambiguidades. Revista Panamericana de Salud Pública, 36(3), 164-170.

Lima, R.C. (2020). Distanciamento e isolamento sociais pela Covid-19 no Brasil: impactos na saúde mental. Physis: Revista de Saúde Coletiva, 30(2), 1-10.

Malta, D. C., Szwarcwald, C. L., Barros, M. B. de A., Gomes, C. S., Machado, I. E., Júnior, P. R. B. de S., Romero, D. E., Lima, M. G., Damacena, G. N., Pina, M. F., Freitas, M. I. F., Werneck, A. O., Silva, D. R. P., Azevedo, L. O., \& Gracie, R. (2020). A pandemia da COVID-19 e as mudanças no estilo de vida dos brasileiros adultos: um estudo transversal, 2020. Epidemiologia e Serviços de Saúde, 29(4), 1-13. 
Research, Society and Development, v. 11, n. 3, e9011326133, 2022

(CC BY 4.0) | ISSN 2525-3409 | DOI: http://dx.doi.org/10.33448/rsd-v11i3.26133

Maqsood, M. B., Islam, M. A., Nisa, Z. U., Naqvi, A. A., Al Qarni, A., Al-Karasneh, A. F., \& Haseeb, A. (2021). Assessment of quality of work life (QWL) among healthcare staff of intensive care unit (ICU) and emergency unit during COVID-19 outbreak using WHOQoL-BREF. Saudi Pharm J, 29(11), 1348-1354. doi:10.1016/j.jsps.2021.09.002

Matsudo, S., Araújo, T., Marsudo, V., Andrade, D., Andrade, E., Oliveira, 1. C., \& Braggion, G. (2001). Questionário Internacional de Atividade Física (IPAQ): estudo de validade e reprodutibilidade no Brasil. Revista Brasileira de Atividade Física \& Saúde, 6(2), 5-18.

Minayo, M.C.S., Hartz, Z.M.A., \& Buss, P.M. (2000). Qualidade de vida e saúde: um debate necessário. Revista Ciência \& Saúde Coletiva, 5(1), 7-18.

Neto, R. B. G. (2020). Impactos da COVID-19 sobre a economia mundial. Boletim de Conjuntura (BOCA), 2(5), 113-127.

Ornell, F., Schuch, J. B., Sordi, A.O., \& Kessle, F. H. P. (2020). Pandemia de medo e COVID-19: impacto na saúde mental e possíveis estratégias. Revista Debates em Psiquiatria, 10(2), 12-17.

Park, K. H., Kim, A. R., Yang, M. A., Lim, S. J., \& Park, J. H. (2021). Impact of the COVID-19 pandemic on the lifestyle, mental health, and quality of life of adults in South Korea. PLoS One, 16(2), e0247970. doi:10.1371/journal.pone.0247970

Pereira, L. O., Francischi, R. P., \& Lancha, A. H. (2003). Obesidade: hábitos nutricionais, sedentarismo e resistência à insulina. Arquivos Brasileiros de Endocrinologia \& Metabologia, 47(2), 111-127.

Pereira, R. J. (2005). Análise da qualidade de vida de idosos - município de Teixeiras-MG. 2005. 159f. Dissertação (Mestrado em Ciência da Nutrição). Universidade Federal de Viçosa, Viçosa.

Pereira, R. J., Cotta R. M. M., Franceschini, S. C.C., Ribeiro, R. C. L., Sampaio, R. F., Priore, S. E., \& Cecon, P. R. (2006). Contribuição dos domínios físico, social, psicológico e ambiental para a qualidade de vida global de idosos. Revista de Psiquiatria do Rio Grande do Sul, Rio Grande do Sul, $28(1), 27-38$.

Polero, P., Rebollo-Seco, C., Adsuar, J. C., Pérez-Gómez, J., Rojo-Ramos, J., Manzano-Redondo, F., \& Carlos-Vivas, J. (2021). Physical Activity Recommendations during COVID-19: Narrative Review. 18(1), 65.

Rajpal, A., Rahimi, L., \& Ismail-Beigi, F. (2020). Factors leading to high morbidity and mortality of COVID-19 in patients with type 2 diabetes. J Diabetes, 12(12), 895-908. 10.1111/1753-0407.13085

Schmidt, B., Crepaldi, M. A., Bolze, S. D. L. A., Silva, L. N., \& Demenech, L. M. (2020). Impactos na Saúde Mental e Intervenções Psicológicas Diante da Pandemia do Novo Coronavírus (COVID-19). Estudos de Psicologia, 37, 1-13.

Silva, I. C., Knuth, A. G., Wendt, A., Nunes B. P., Hallal, P. C., Santos, L. P., Harter, J., \& Pellegrini, D. C. P. (2020). Prática de atividade física em meio à pandemia da COVID-19: estudo de base populacional em cidade do sul do Brasil. Revista Ciência \& Saúde Coletiva, 25(11), 4249-4258.

Silva, R. S., Silva, I., Silva, R. A., Souza, L., \& Tomasi, E. (2010). Atividade física e qualidade de vida. Revista Ciência \& Saúde Coletiva,15(1), 115-120.

Tonin, L., Lacerda, M. R., Caceres, N. T. G., \& Hermann, A. P. (2020). Recomendações em tempos de COVID-19: um olhar para o cuidado domiciliar. Revista Brasileira de Enfermagem, 73, 1-5.

World Health Organization. (2020) Director-General's opening remarks at the media briefing on COVID-19.: https://www.who.int/emergencies/diseases/novelcoronavirus-2019. 